\title{
Finite Ground Coplanar Waveguide (FGC) Low Loss, Low Coupling 90-Degree Crossover Junctions
}

\author{
George E. Ponchak, Senior Member, IEEE, and Emmanouil M. Tentzeris, Member, IEEE
}

\begin{abstract}
Microwave and millimeter-wave integrated circuits and RF distribution networks often require two transmission lines to cross over each other. In this paper, experimental measurements and three-dimensional (3-D) finite difference time domain analysis are used to thoroughly characterize coplanar waveguide (CPW) and finite ground coplanar waveguide (FGC) 90-degree crossover junctions. It is shown that FGC crossover junctions have approximately 15 dB lower coupling than CPW crossover junctions. Furthermore, it is shown that the FGC junctions do not excite the parasitic slotline mode, whereas, the CPW junctions do excite the slotline mode. The results presented indicate that the FGC crossover junction is easier to implement and has better characteristics than the CPW crossover junction.
\end{abstract}

Index Terms-Coplanar waveguide, coupling, finite ground coplanar waveguide, planar transmission lines, transmission lines.

\section{INTRODUCTION}

$\mathbf{M}$ ONOLITHIC microwave and millimeter-wave integrated circuits (MMICs), microwave multichip modules (MCMs), and antenna distribution networks often require two transmission lines to cross over each other as shown schematically in Fig. 1. These crossover junctions are straight forward to implement with a single airbridge for circuits based on microstrip transmission lines. However, circuit designers often prefer coplanar waveguide (CPW) transmission lines because it is a planar transmission line, which enables easy series and shunt element connections without metal filled via holes [1]. This simplifies the fabrication process, eliminates backside processing, and lowers fabrication cost by approximately $30 \%$. Recently, an improved version of CPW has been developed called Finite Ground Coplanar waveguide (FGC). FGC has electrically narrow ground planes that have been shown to reduce parasitic resonances caused by parallel plate modes, enable novel uses of the ground planes to integrate lumped elements, reduce circuit size, and reduce coupling between parallel transmission lines [2]-[5]. However, only preliminary results on CPW and FGC crossover junctions have been presented [6].

In this paper, experimental measurements and a three-dimensional (3-D) finite difference time domain (3-D-FDTD) analysis are used to characterize CPW and FGC crossover junctions. First, the crossover junction is described in detail, fol-

Manuscript received June 16, 2000; revised August 7, 2002. This work was presented in part at the IEEE MTT-S International Microwave Symposium, Boston, MA, June 11-16, 2000.

G. E. Ponchak is with the Electron Device Technology Branch, NASA Glenn Research Center, Cleveland, OH 44135 USA.

E. M. Tentzeris is with the School of Electrical and Computer Engineering, Georgia Institute of Technology, Atlanta, GA 30332-0250 USA.

Digital Object Identifier 10.1109/TADVP.2002.805314

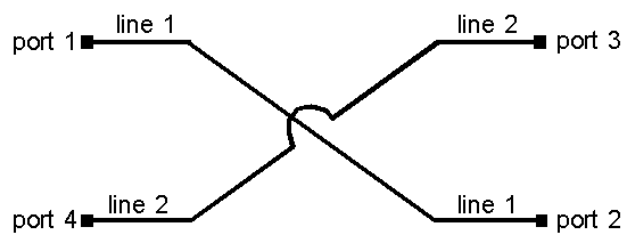

Fig. 1. Schematic of $90^{\circ}$ crossover junction.

lowed by the experimental and theoretical procedures. Then, the scattering parameters of the 4-port junction are presented with a discussion of their significance and the impact of coupling between the CPW and the slotline modes. Finally, electric field plots and 3-D-FDTD results are used to physically show why the FGC crossover junctions are superior to $\mathrm{CPW}$ crossover junctions.

\section{CirCUIT DESCRIPTION}

Both CPW and FGC crossover junctions require the center strip of one line to cross over the second line; however, the ground plane connections are different. For CPW discontinuities, it is known that the ground planes of each CPW line must be tied together by airbridges at each discontinuity to short out the parasitic slotline mode that is easily excited. Since CPW ground planes are very wide, it is impossible to fabricate an airbridge that would enable the ground planes of one CPW line to completely cross over the second CPW line as the center strip does. Thus, at the CPW crossover junction, the two lines necessarily share their ground planes. Two CPW crossover junction layouts are possible. The first is shown in Fig. 2(a), CPW1, and it uses underpasses to connect two of the four ground planes at the junction reference planes. Because the center strips are now further apart, the airbridge connecting them is long as seen in Fig. 2(a). The second layout, CPW2, is shown in Fig. 2(b). It uses airbridges to connect all of the ground planes together, but two of the ground plane airbridges are set back from the junction $25 \mu \mathrm{m}$ to ensure that they do not interfere with the center strip airbridge during processing.

The ground planes of FGC are both electrically and physically narrow, which enables airbridges to be used for the ground planes as well as the center strip. Therefore, FGC lines may cross over each other without sharing any metal structures between them by employing three airbridges. Each of these airbridges is longer than those used for the CPW junctions, but because FGC is a narrow transmission line, they are not too long and do not collapse. Fig. 3 shows an SEM photograph of the FGC crossover junction, FGC1. Note that FGC1 is a simple crossover junction with airbridges of the same width as 


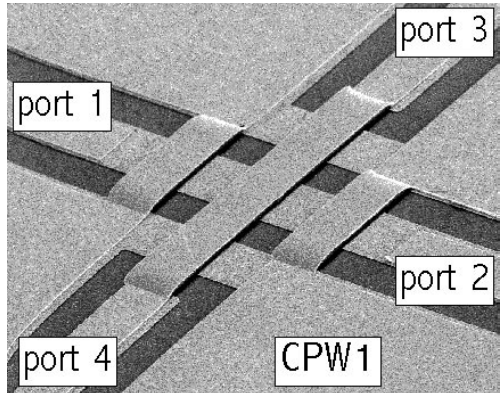

(a)

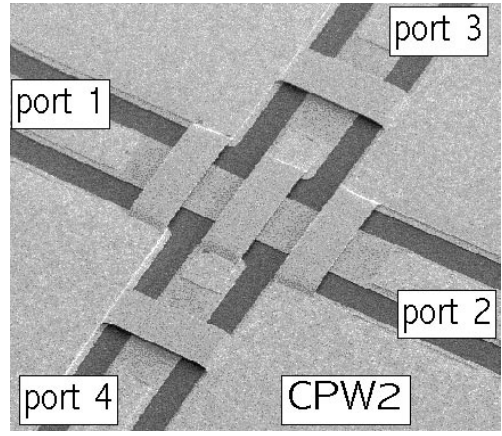

(b)

Fig. 2. SEM micrograph of coplanar waveguide (CPW) junction. (a) CPW1 has ground planes connected by underpasses. (b) CPW2 has all ground planes connected by airbridges.

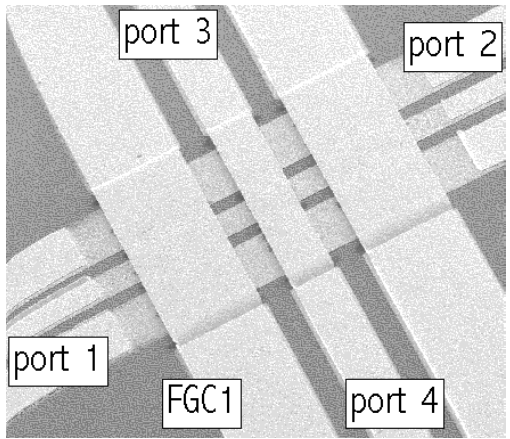

Fig. 3. SEM micrograph of finite ground coplanar waveguide (FGC) crossover junction. Both lines have the same dimensions.

the center strip and the ground planes. Although not shown, variations of FGC1 have also been characterized. FGC2 is similar to FGC1, but the ground plane airbridges are one half the width while the other dimensions, including the slot width, are held constant. FGC3 is similar to FGC2, but the first level metal defining the ground strips under the airbridges is one half the width.

\section{EXPERIMENTAL PROCEDURE}

Two sets of circuits are fabricated on Si wafers with a resistivity of $2500 \Omega-\mathrm{cm}, \varepsilon_{r}=11.7$, and a thickness of $410 \mu \mathrm{m}$. Prior to fabrication, each wafer is thoroughly cleaned, including an $\mathrm{HF}$ acid dip to remove the native oxide. For the first set, the first level metal is defined by a lift-off process and consists of $0.02 \mu \mathrm{m}$ of Ti and $0.6 \mu \mathrm{m}$ of Au. A $1.0 \mu \mathrm{m}$ Au plating process is then used to build the center strip, the ground planes, and the airbridges, which results in the center conductors and ground

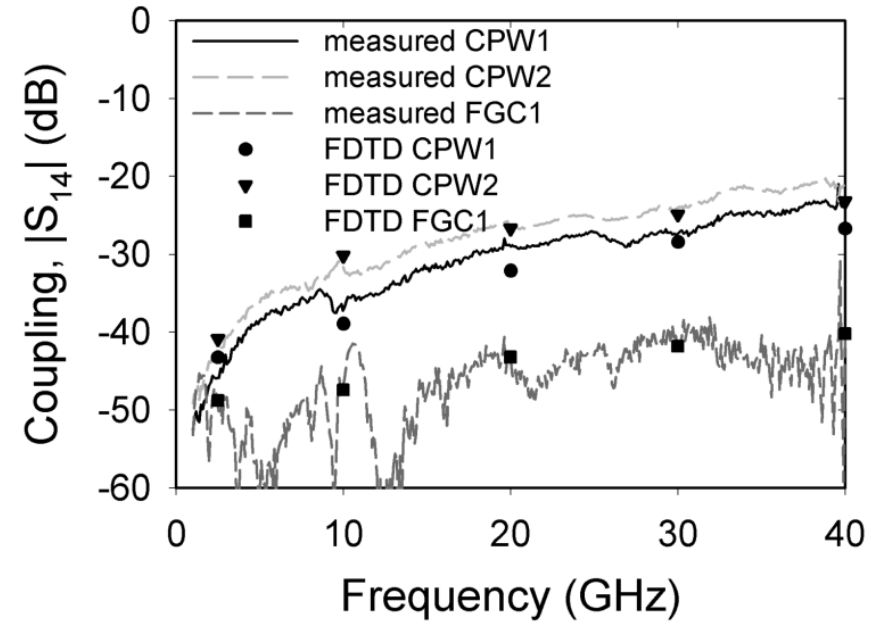

Fig. 4. Measured and FDTD calculated coupling between CPW and FGC crossover junctions from set 1 .

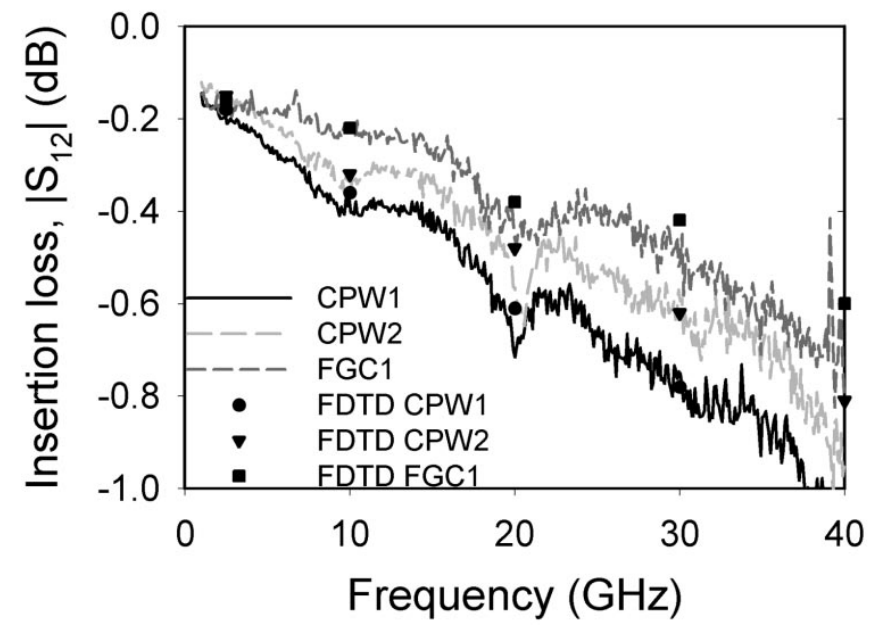

Fig. 5. Measured and FDTD calculated insertion loss of CPW and FGC crossover junctions from set 1 .

planes having a total thickness of $1.6 \mu \mathrm{m}$ and the airbridges having a total Au thickness of $1.0 \mu \mathrm{m}$. The airbridge height above the Si substrate is approximately $3 \mu \mathrm{m}$, or $2.4 \mu \mathrm{m}$ above the first level metal. No insulators, $\mathrm{SiO}_{2}$ or $\mathrm{Si}_{3} \mathrm{~N}_{4}$, are grown on the wafers either before or after metallization for passivation. The second set of circuits was processed similarly, but the first level metal is $1.2-\mu \mathrm{m}$ thick, CPW/FGC final metal thickness is $2.5 \mu \mathrm{m}$, and the airbridge height is $4.0 \mu \mathrm{m}$ above the $\mathrm{Si}$ or $2.8 \mu \mathrm{m}$ above the first level metal.

The CPW lines have a center strip width, $S$, and slot width, $W$, of 30 and $22 \mu \mathrm{m}$, respectively. The CPW ground plane is $150-\mu \mathrm{m}$ wide, which, at five times the center strip width, is equivalent to an infinite width ground plane [2]. $S$ and $W$ of the FGC lines are 32 and $19 \mu \mathrm{m}$, respectively, and the ground plane width, $B$, is $64 \mu \mathrm{m}$. These lines are designed to have similar $S+2 W$ and a nominal characteristic impedance of $50 \Omega$ so that their characteristics can be compared. Besides the airbridges at the crossover junction, an airbridge is located immediately after the probe pads to short the parasitic slotline mode that is often excited at CPW junctions. 


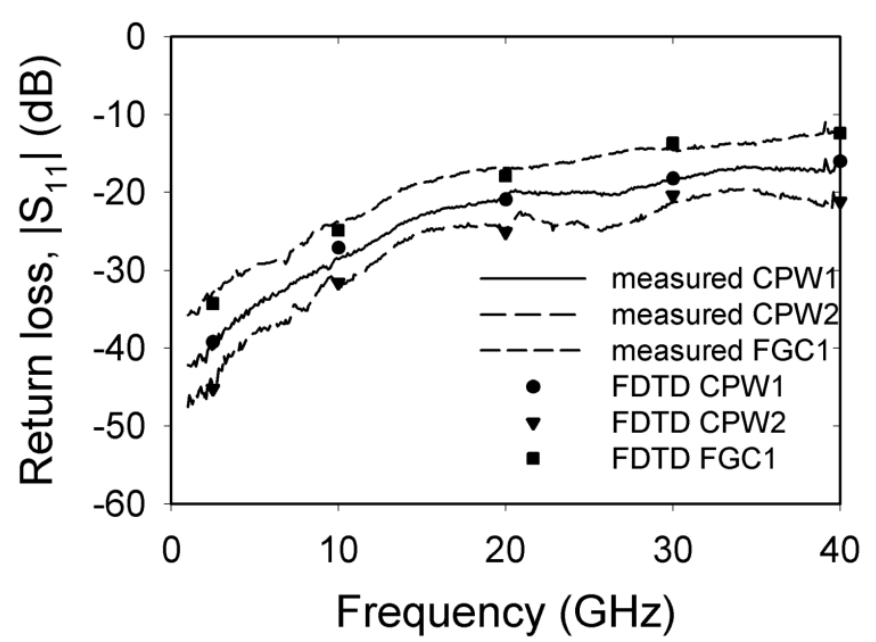

Fig. 6. Measured and FDTD calculated return loss for CPW and FGC crossover junctions from set 1 .

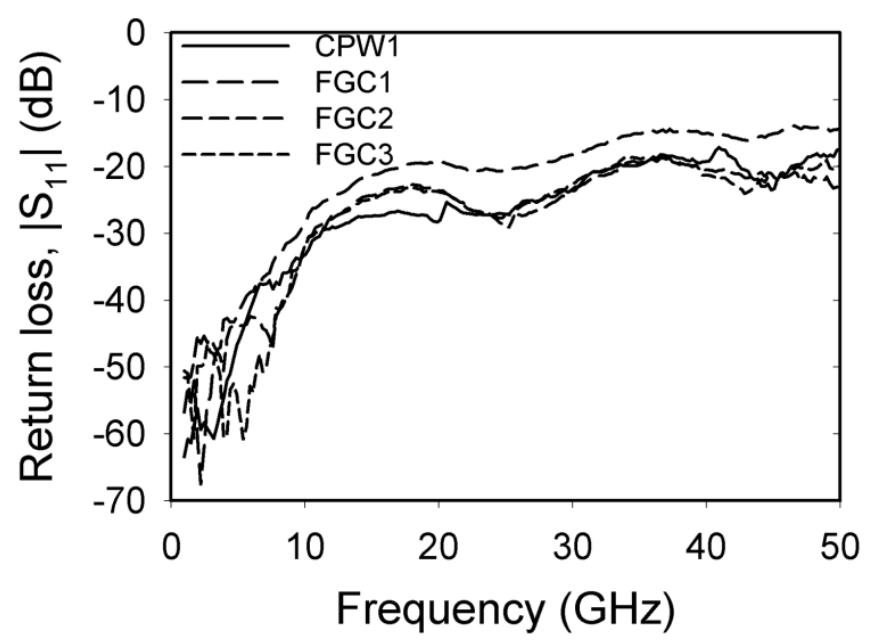

(a)

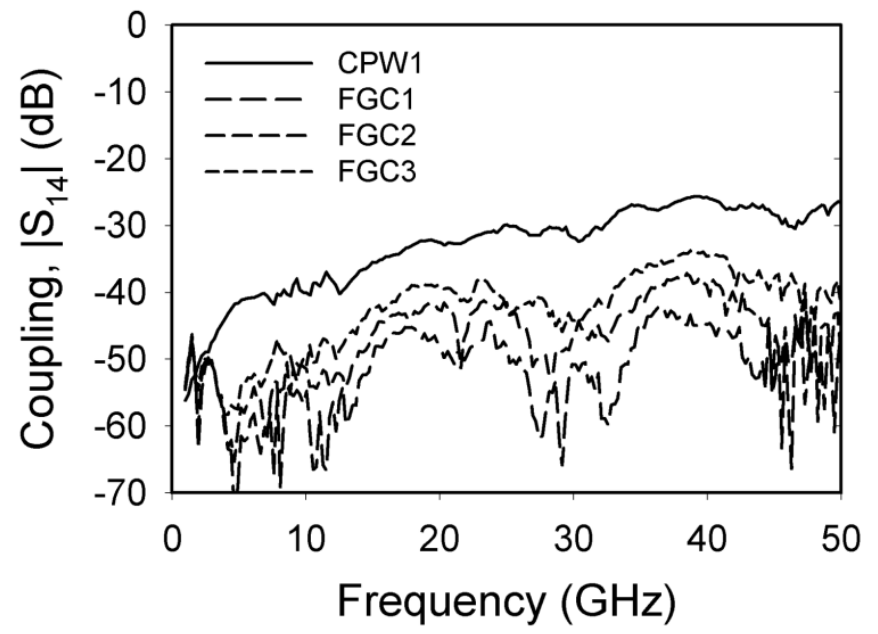

(b)

Fig. 7. (a) Measured return loss for $\mathrm{CPW}$ and $\mathrm{FGC}$ crossover junctions from set 2. (b) Measured coupling for CPW and FGC crossover junctions from set 2.

A thru-reflect-line (TRL) calibration is implemented through the MULTICAL software [7] routine, with the calibration standards fabricated on the same wafer as the test circuits. Thus, the reference plane is accurately placed at the crossover junction.

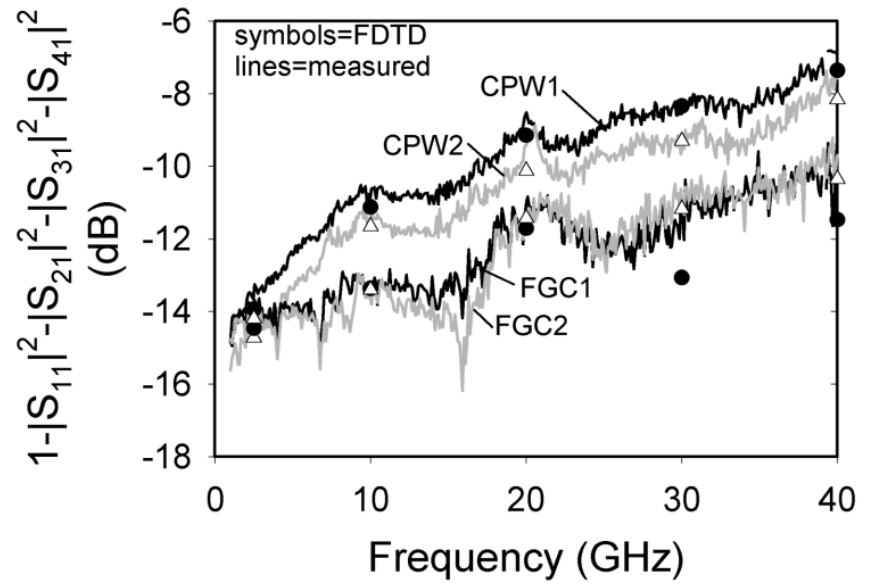

Fig. 8. Measured and FDTD calculated power loss for CPW and FGC crossover junctions from set 1 .

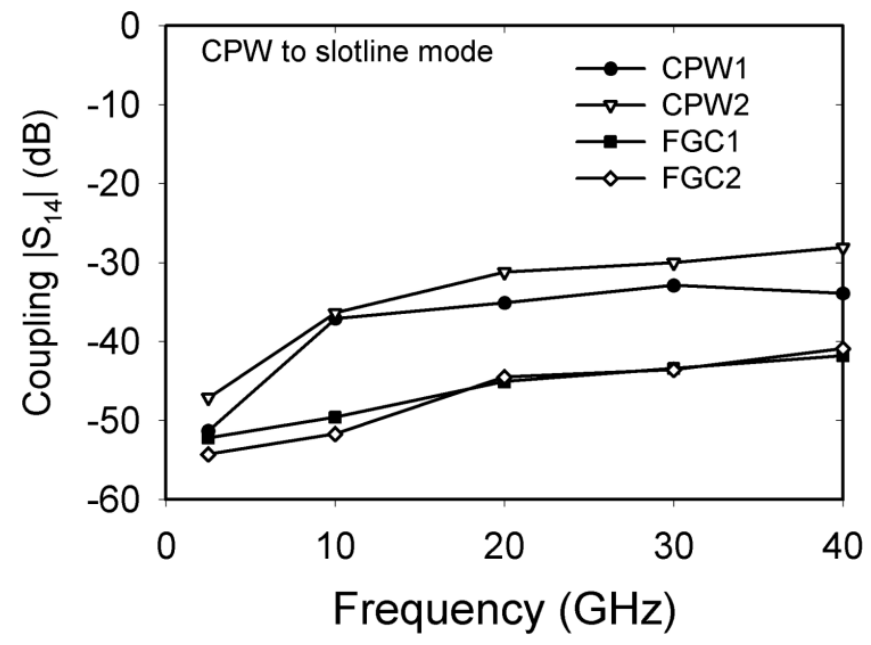

Fig. 9. FDTD calculated slotline mode at the coupled ports.

To measure the 4-port scattering parameters, a HP vector network analyzer, GGB Industries picoprobes, and a quartz wafer between the Si wafer and the metal wafer chuck is used. Since the network analyzer only measures two port circuits, two of the four ports are terminated by specially designed GGB picoprobes with built in $50 \Omega$ terminations.

\section{THEORETICAL MODELING (FDTD)}

Theoretical characterization of the coupling between the lines at the crossover junctions is obtained through the 3-D-FDTD method [2], [8]. In the theoretical analysis, the 3-D junction with nonzero conductor thicknesses is used, but the conductors and dielectrics are all assumed to be lossless. Thus, the FDTD results only include power lost to radiation and to non-CPW modes. The FDTD is implemented with: interleaved positioning of the electric and magnetic field components to provide a second-order accuracy of the algorithm; grids of 54 by 200 by 80 cells terminated with 4 Perfectly Matched Layers (PML) cells in each direction to provide accurate results for a time-step $\Delta t=0.9 \Delta t_{\max }$; and the superposition of the excitation on the FDTD calculated field value of all cells of the 


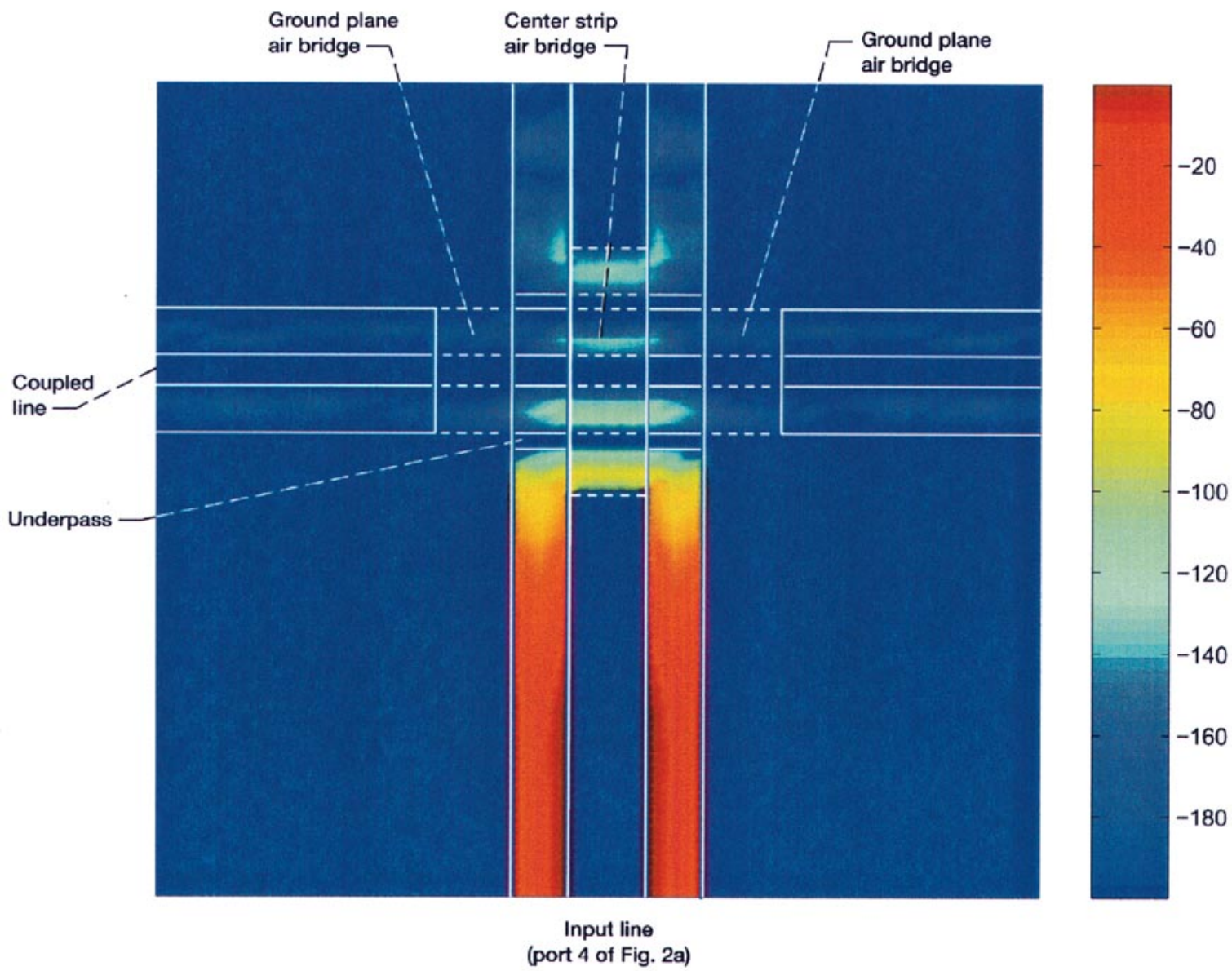

Fig. 10. (a) FDTD calculated electric fields in the plane of the substrate for CPW1 after 700 time steps.

excitation region for each time-step to guarantee the elimination of spurious retroreflective effects (total field formulation). A Gaussian pulse with $f_{\max }=40 \mathrm{GHz}$ and odd horizontal spatial distribution is used to excite only the CPW mode in the circuit; however, the CPW mode and all parasitic modes that are generated by the junctions are modeled. To achieve a faster convergence (less than 15000 time-steps) the Gaussian pulse is multiplied by a correction spatial factor that accounts for the edge effects of the ground and signal conductors. Two probes placed symmetrically on either side of the center conductor in the slot regions are used for the decomposition of the field into CPW and slotline modes. Finally, the frequency-domain results are derived from the time-domain values through the application of FFT algorithm.

\section{RESUlTS}

The measured and calculated coupling, $\left|S_{41}\right|$, insertion loss, $\left|S_{21}\right|$, and return loss, $\left|S_{11}\right|$, for CPW1, CPW2, and FGC1 from the first set of circuits is shown in Figs. 4-6, respectively. It is seen that the measured and calculated results are in excellent agreement across the entire frequency range, which indicates a high level of accuracy in the characterization methods. Recall that the FDTD results do not include conductor and dielectric losses whereas the experimental results include all loss mechanisms; therefore, the excellent agreement between theoretical and measured results indicates that conductor and dielectric losses play a minor role in the junction characteristics. In fact, measured attenuation for CPW and FGC lines from sets 1 and 2 is approximately $5.2 \mathrm{~dB} / \mathrm{cm}$ and $3.3 \mathrm{~dB} / \mathrm{cm}$ at $40 \mathrm{GHz}$ respectively. Using this measured attenuation, a CPW line the length of the junction, $S+2 W$, should have $0.04 \mathrm{~dB}$ of loss for set 1 and $0.025 \mathrm{~dB}$ of loss for set 2 at $40 \mathrm{GHz}$. Similarly, a FGC line the length of the junction, $S+2 W+2 B$, should have a loss of $0.1 \mathrm{~dB}$ for set 1 and $0.065 \mathrm{~dB}$ for set 2 at $40 \mathrm{GHz}$. Comparing these values to those shown on Fig. 5 clearly indicates the small role that conductor and dielectric losses play in the junction's characteristics.

Comparing the two CPW junctions shows that CPW1 has approximately $2.5 \mathrm{~dB}$ lower coupling, but it has higher insertion loss and lower return loss; however, the difference between the characteristics of the two CPW junctions is minor and either may be used in practice. More importantly, Fig. 4 shows that the FGC1 crossover junction has approximately $15 \mathrm{~dB}$ lower coupling than either CPW crossover junction across the entire frequency band. In addition, FGC1 has lower insertion loss than the CPW junctions as shown in Fig. 5, but $\left|S_{11}\right|$ of the FGC1 crossover junction is approximately $5 \mathrm{~dB}$ higher. Measured characteristics of junctions from the second set of circuits are similar, but the insertion loss is lower due to the thicker gold lines and the coupling of FGC1 is lower due to the higher airbridges. 


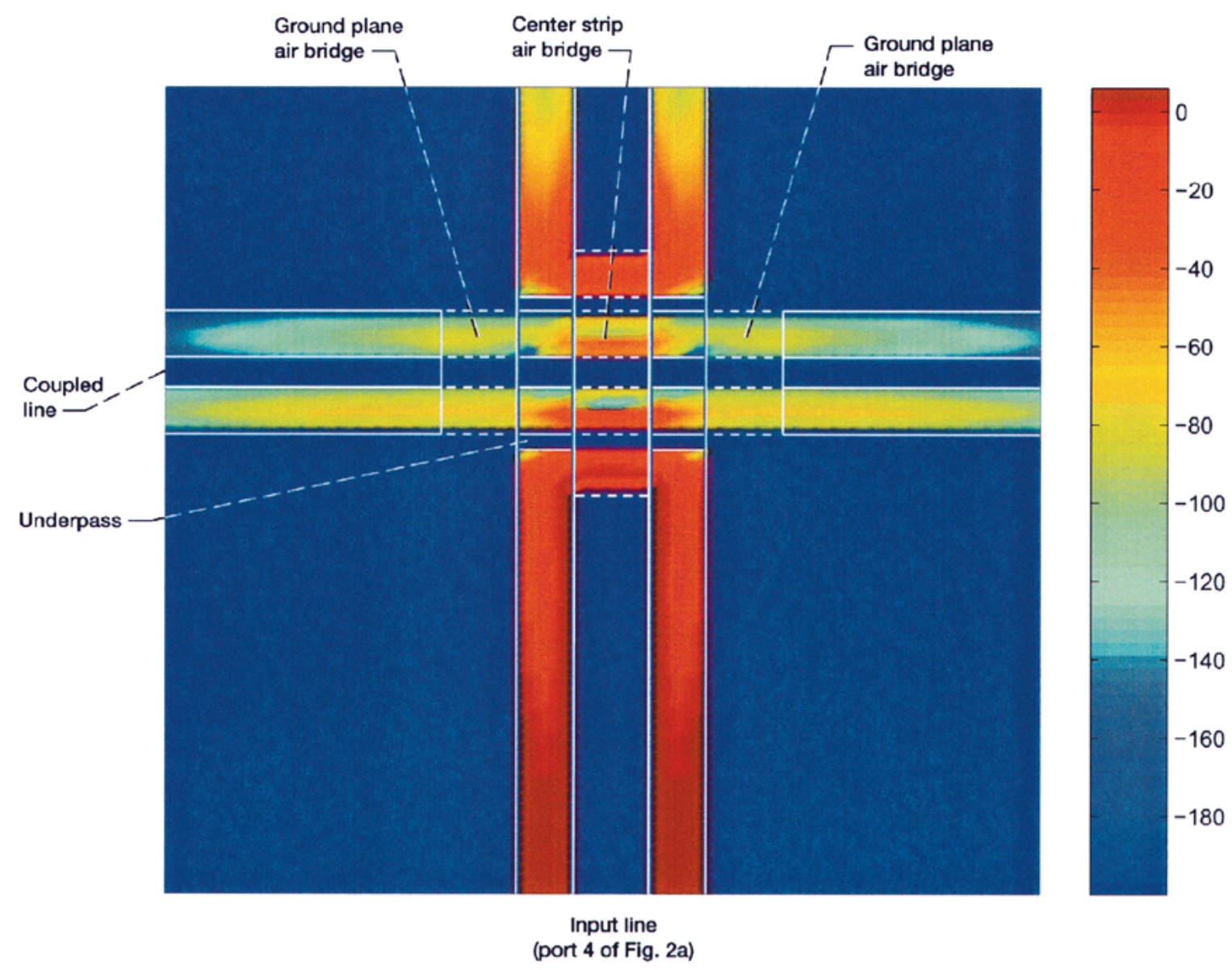

Fig. 10. (Continued) (b) FDTD calculated electric fields in the plane of the substrate for CPW1 after 900 time steps.

Although the FGC1 crossover junction has good characteristics, the return loss is too low for some applications. Since the two FGC lines at the crossover junction do not share any metal structures between them, the junction characteristics, in particular the impedance mismatch that causes high reflections, must be dominated by capacitance. Therefore, FGC2 and FGC3 are developed. FGC2, with half the ground strip airbridge width, should have half the capacitance of FGC1, and FGC3, with both the upper and lower ground strips being half the width at the junction, should have one forth the capacitance as FGC1. The measured return loss and coupling for FGC1, FGC2, and FGC3 from the second set of circuits is shown in Fig. 7(a) and (b), respectively, with the characteristics of CPW1 included for comparison. As shown, the return loss of the FGC crossover junction improves with the narrowing of the ground plane strips from 15 to $20 \mathrm{~dB}$ at $40 \mathrm{GHz}$, and the FGC2 and FGC3 junctions now have the same return loss as the CPW1 junction. However, a slight penalty is paid since the measured coupling of FGC2 and FGC3 is approximately 5 and $8 \mathrm{~dB}$ higher across the frequency band respectively. It must be noted that measured characteristics from the first set of circuits and the FDTD results show a much smaller increase in coupling, approximately $2 \mathrm{~dB}$, while the improvement in return loss is similar to that shown in Fig. 7(a). The increase in coupling for FGC2 and FGC3 compared to FGC1 indicates that significant capacitance is generated at the junction when the ground plane is narrowed from $2 S$ to $S$. Electric fields generated by the narrowing of the ground plane terminate on the second FGC line and thus increase coupling. However, the lower distributed capacitance due to the narrower ground strips helps maintain the characteristic impedance of the FGC line and thus lower $\left|S_{11}\right|$.

Even though it has been shown that FGC crossover junctions have lower insertion loss than CPW crossover junctions, the character of that loss has not been addressed. First, the magnitude of the power lost needs to be established by calculating $1-\left|S_{11}\right|^{2}-\left|S_{21}\right|^{2}-\left|S_{31}\right|^{2}-\left|S_{41}\right|^{2}$, which should equal 0 for a lossless junction [9]. Fig. 8 shows the theoretical and measured power loss for junctions from set 1 . It is seen that CPW1 has less power loss than CPW2, but again the difference (approximately $1 \mathrm{~dB}$ ) between the two CPW junctions is small. FGC1 and FGC2 have nearly equal characteristics with a total power loss that is approximately $3 \mathrm{~dB}$ less than the $\mathrm{CPW}$ junctions at $40 \mathrm{GHz}$. FGC3 is not shown for clarity, but it has the same characteristics as FGC1 and FGC2. Characteristics from the second set of circuits, not shown, are in agreement with these results. Since it has been established that conductor and dielectric loss is not significant for the crossover junction, radiation is usually assumed to be responsible for the remaining loss. However, power lost to the slotline mode that is excited at the junction is another possibility. Note that the airbridges placed at the probe pads and the use of Ground-Signal-Ground probes eliminates the measurement of power in the slotline mode in 


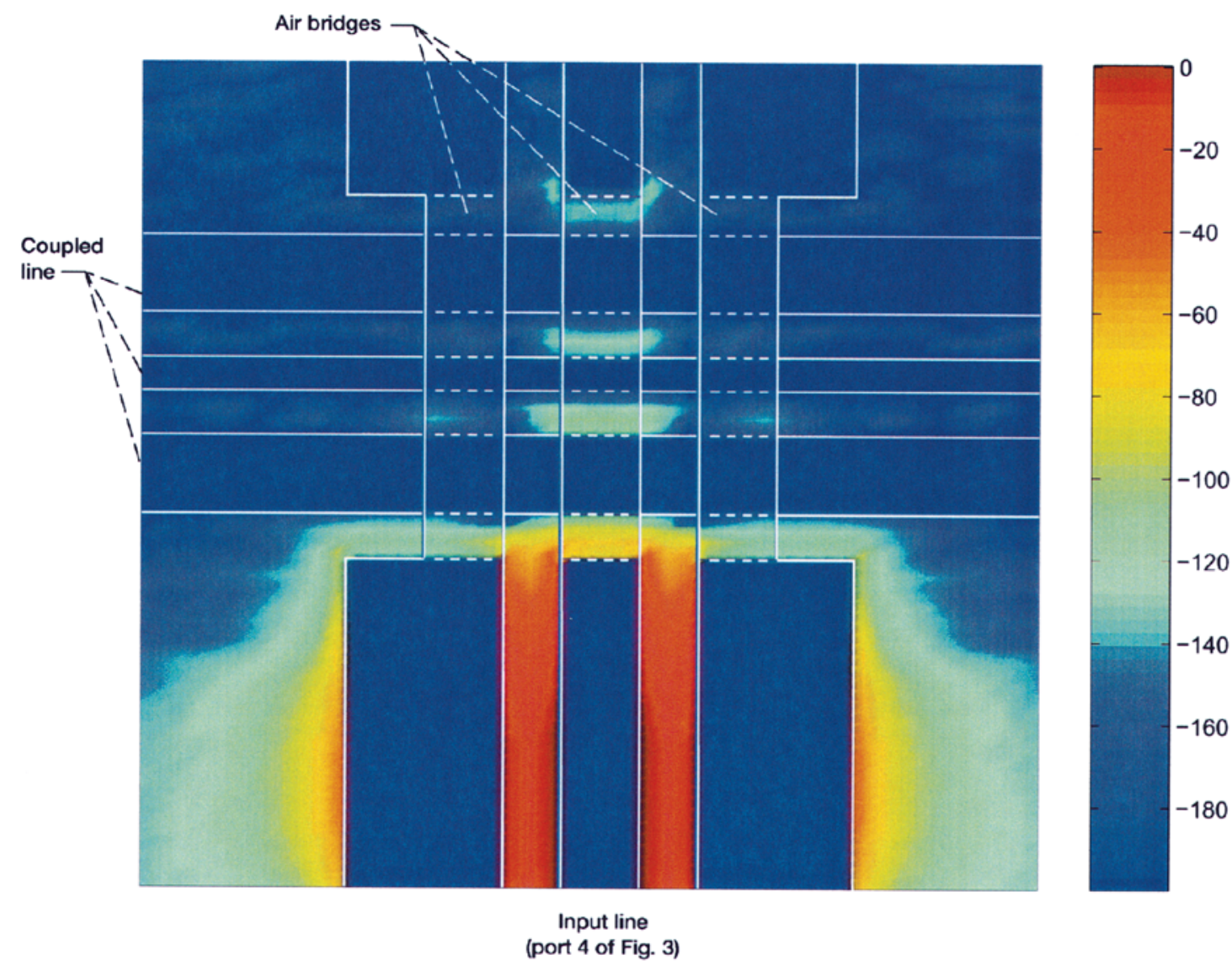

Fig. 11. (a) FDTD calculated electric fields in the plane of the substrate for FGC2 after 700 time steps.

the experimental data, but the FDTD analysis can differentiate the slotline modes from the CPW modes. Fig. 9 shows the slotline mode in the coupled ports, $\left|S_{31}\right|$ and $\left|S_{41}\right|$, for each of the four junctions. The two FGC crossover junctions have very low excitation of the slotline mode, less than $40 \mathrm{~dB}$, while the $\mathrm{CPW}$ crossover junctions have higher excitation of the slotline modes, approximately $30 \mathrm{~dB}$. Excitation of the slotline modes at ports 1 and 2 is calculated to be less than $60 \mathrm{~dB}$ for all of the junctions. Thus, if the power loss for each junction shown in Fig. 8 is corrected for the conductor loss and the slotline mode coupling, the CPW and FGC crossover junctions have an unaccounted power loss of $8.45 \mathrm{~dB}$ and $12.5 \mathrm{~dB}$ at $40 \mathrm{GHz}$, respectively. This remaining loss is due to excitation of surface wave modes and free space radiation.

Typically, airbridges are required at all CPW junctions to suppress excitation of the slotline mode, but as just shown, this is not necessary for the FGC crossover junctions. However, if added security against the slotline mode is required, two airbridges connecting the two ground strips of line 1 and two airbridges connecting the ground strips of line 2 may be added to the FGC lines. A set of FGC crossover junctions were fabricated and characterized with these extra airbridges. While the measured coupling does not change from the results shown in Fig. $4,\left|S_{11}\right|$ increases by approximately $2 \mathrm{~dB}$ across the entire frequency range. Thus, airbridges connecting the ground planes of each FGC line is not required for this circuit.

\section{EleCtromagnetic FieldS AT JunCtion}

While the previous sections have shown that FGC crossover junctions have lower coupling and power loss to slotline modes than CPW crossover junctions, the reason for these characteristics has not been addressed. These issues will be addressed in this section by employing the 3-D-FDTD analysis.

First, the crossover junction CPW1 is analyzed. Fig. 10(a) and (b) show the magnitude of the electric fields on the plane of the Si substrate after 700 and 900 time steps, respectively. The metalized areas of the substrate that comprise the CPW structure have no tangential electric field components because perfect conductors are assumed; thus, they appear as dark blue. At 700 time steps, see Fig. 10(a), the incident electric fields has just arrived at the crossover junction as evidenced by the excitation of electric fields in the lower slot of the coupled CPW line. At 900 time steps, the incident electric fields have traversed the junction as shown in Fig. 10(b). In Fig. 10(b), it is seen that $|E|$ in the lower slot of the coupled CPW line is higher than in the upper slot. In addition, the $E$ excited in the slots of the coupled line are out of phase; the leading edge of the $E$ in the lower slot is ahead of the leading edge of the $E$ in the upper slot. This is clear evidence of the excitation of the slotline mode in the coupled CPW line. Fig. 10(b) also shows that the $E$ in the input and output line is equal in magnitude and phase, which indicates the slotline mode is not excited in the input line. Furthermore, because all of the current flowing in the ground planes of the input line 


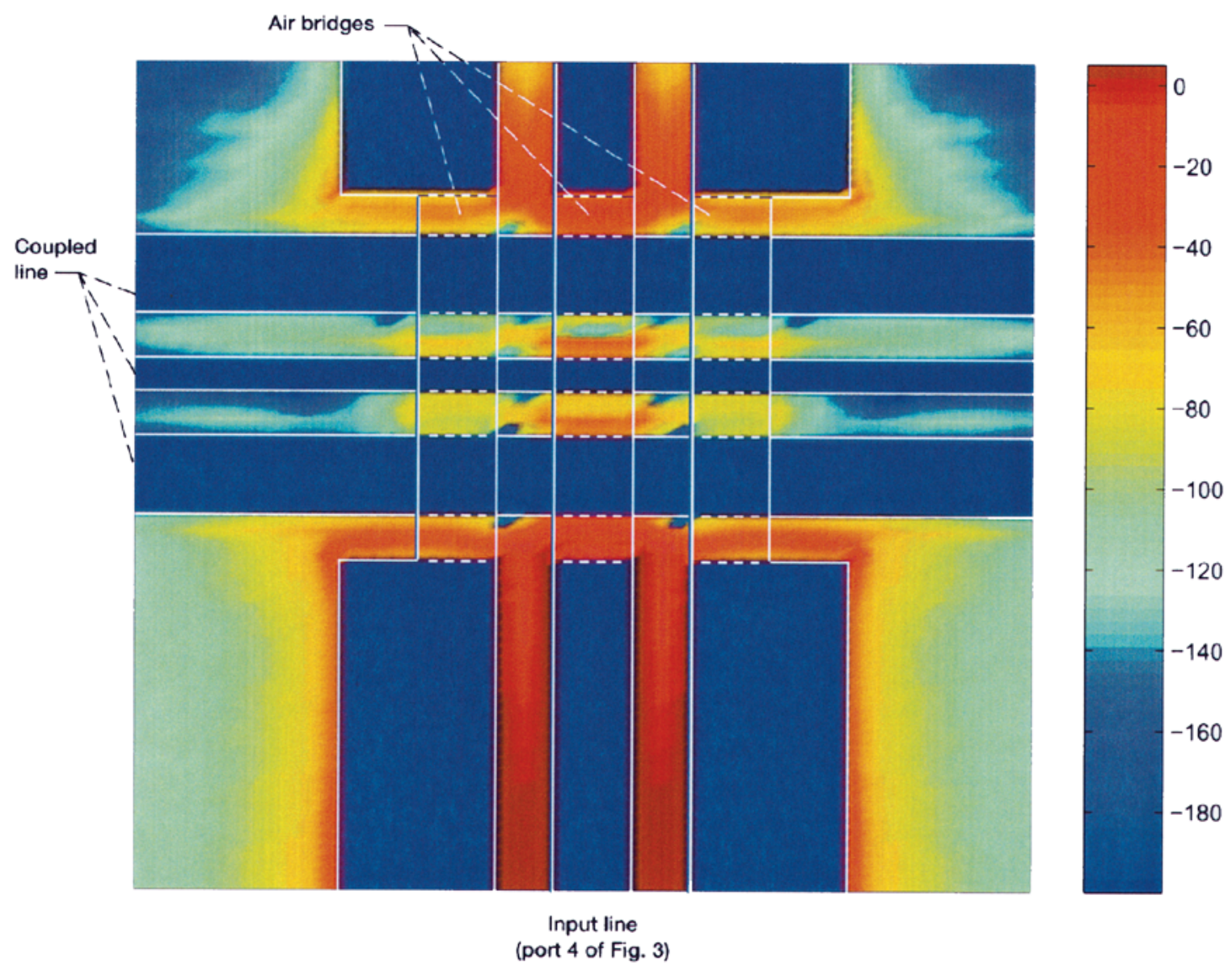

Fig. 11. (Continued) (b) FDTD calculated electric fields in the plane of the substrate for FGC2 after 900 time steps.

does not flow through the ground plane airbridges, the current splits at the junction with most of the current flowing through the airbridges while some current flows along the ground plane of the coupled line, the sum of the currents in the two ground plane airbridges do not balance the current flowing in the center strip airbridge. Thus, the currents excited in the coupled CPW line by the center strip airbridge of the input line are not canceled by the currents excited by the ground plane airbridges and the coupling and slotline mode excitation are high. Further insight is obtained by repeating the modeling with all of the airbridges removed. Ideally, the junction appears as an open circuit at the input port and all of the power is reflected. However, modeling shows that the current that flows through the underpass metal at the end of the input line, see port 4 of Fig. 2(a), excites a current along the slot of the coupled line. In other words, even if the airbridges that create the crossover junction are removed, there would still be coupling between the CPW lines. Thus, coupling is high for the CPW crossover junctions because they share common ground planes and the current in those ground planes cannot be directed through the ground plane airbridges.

Similar electric field plots for the FGC2 crossover junction at 700 and 900 time steps are shown in Fig. 11(a) and (b), respectively. Again, in the 700 time step plot, the incident field has just arrived at the junction. The high electric field on the outer edge of the ground strips of the FGC line are part of the propagating mode for the FGC line as described in [2] and are not due to the junction. At 900 time steps, the electric field has traversed the junction. In comparison to the CPW1 junction shown in Fig. 10(b), the magnitude and phase of the $E$ in the two slots of the coupled line are nearly equal, which indicates lower power in the slotline mode. Furthermore, because most, if not all, of the current in the ground plane strips flows through the ground strip airbridges, the current excited in the coupled FGC line by the center strip airbridge is canceled by the currents excited by the ground strips. Note that while the $|E|$ in the coupled FGC line is high immediately under the junction, the $|E|$ is low outside of the junction. If all of the airbridges are removed and the junction remodeled, it is seen that no current is excited in the coupled line. Thus, the coupling in the FGC crossover junction is lower because no metal structures are shared and the current in the ground planes is more predictably directed.

\section{CONCLUSION}

CPW and FGC crossover junctions have been thoroughly characterized by experimental measurements and a 3-D-FDTD analysis. It is shown that FGC crossover junctions have significantly lower coupling than CPW crossover junctions; the coupling of FGC junctions is less than $40 \mathrm{~dB}$. The return loss of the FGC crossover junction is less than $15 \mathrm{~dB}$, but this may be improved by reducing the width of the ground plane airbridges to achieve $20 \mathrm{~dB}$ return loss. FGC crossover junctions also have very low excitation of the parasitic slotline mode, which makes it unnecessary to add airbridges to connect the ground planes 
of each FGC line. Thus, FGC junctions are easier to implement and have better characteristics than CPW crossover junctions.

\section{REFERENCES}

[1] C. P. Wen, "Coplanar waveguide: A surface strip transmission line suitable for nonreciprocal gyromagnetic device applications," IEEE Trans. Microwave Theory Tech., vol. 17, pp. 1087-1090, 1969.

[2] G. E. Ponchak, E. M. Tentzeris, and L. P. B. Katehi, "Characterization of finite ground coplanar waveguide with narrow ground planes," Int. J. Microcircuits Electron. Packag., vol. 20, no. 2, pp. 167-173, 1997.

[3] G. E. Ponchak and L. P. B. Katehi, "Characteristics of finite ground coplanar waveguide lumped elements," in 1997 IEEE MTT-S Int. Microwave Symp. Dig., Denver, CO, June 8-13, 1997, pp. 1003-1006.

[4] G. E. Ponchak, E. M. Tentzeris, and L. P. B. Katehi, "Characterization of the coupling between adjacent finite ground coplanar (FGC) waveguides," Int. J. Microcircuits Electron. Packag., vol. 20, no. 4, pp. 587-592, Fourth Quarter 1997.

[5] G. E. Ponchak and L. P. B. Katehi, "Finite ground coplanar (FGC) waveguide: A better transmission line for microwave circuits," Adv. Microelectron., pp. 15-18, May/June 1998.

[6] G. E. Ponchak and E. Tentzeris, "Development of finite ground coplanar (FGC) waveguide 90 degree crossover junctions with low coupling," in Proc. 2000 IEEE MTT-S Int. Microwave Symp. Dig., Boston, MA, June 11-16, 2000, pp. 1891-1894.

[7] R. B. Marks, "A multiline method of network analyzer calibration," IEEE Trans. Microwave Theory Tech., vol. 39, pp. 1205-1215, July 1991.

[8] A. Taflove, Computational Electrodynamics. Dedham, MA: Artech House, 1995.

[9] R. E. Collin, Foundations for Microwave Engineering. New York: McGraw-Hill, 1966, p. 174.

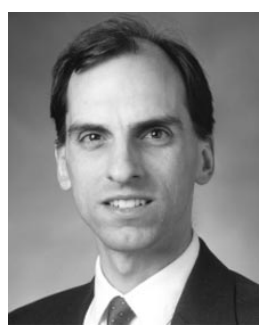

George E. Ponchak (S'82-M'83-SM'97) received the B.E.E. degree from Cleveland State University, Cleveland, OH, in 1983, the M.S.E.E. degree from Case Western Reserve University, Cleveland, $\mathrm{OH}$, in 1987, and the Ph.D. degree from the University of Michigan, Ann Arbor, in 1997.

In July 1983, he joined the staff of the Communication Technology Division, NASA Glenn Research Center, Cleveland, where he is now a Senior Research Engineer. He is interested in the development and characterization of microwave and millimeter-wave printed transmission lines and passive circuits, multilayer interconnects, uniplanar circuits, microwave microelectromechanical (MEMS) components, and microwave packaging. He is responsible for the development of GaAs, InP, and SiGe MMIC's and reliability characterization of them for space applications. He is the author and co-author of more than 80 papers in referred journals and symposia proceedings. From 1997 to 1998 and from 2000 to 2001, he was a Visiting Lecturer at Case Western Reserve University.

Dr. Ponchak received the Best Paper of the ISHM'97 30th International Symposium on Microelectronics Award. He is a Member of the IEEE MTT-S Society and the International Microelectronics and Packaging Society (IMAPS).

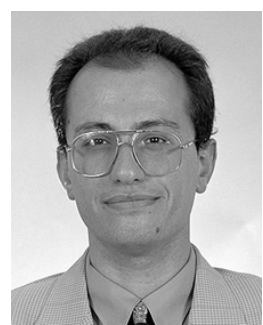

Emmanouil M. Tentzeris (M'99) received the Diploma degree in electrical engineering and computer science (with high honors) from the National Technical University, Athens, Greece, in 1992 and the M.S. and Ph.D. degrees in electrical engineering and computer science from the University of Michigan, Ann Arbor, in 1993 and 1998, respectively.

He joined the School of Electrical and Computer Engineering, George Institute of Technology (Georgia Tech), Atlanta, in 1998 as an Assistant Professor and he is currently the Leader of the Novel Integration Techniques Subthrust, Broadband Hardware Access Thrust, Yamacraw Initiative, State of Georgia, and the Packaging Research Center thrust Leader for RF/Wireless Packaging. At Georgia Tech, he has established academic programs in numerical electromagnetics, RF/wireless applications, packaging adaptive ultracompact antennas and RF MEMS modeling: over 75 publications, three book chapters, and numerous invited papers. He has been a Visiting Professor at the Technical University of Muenchen, Munich, Germany for the summer of 2002.

Dr. Tentzeris received the National Science Foundation CAREER Award (2000), the Georgia Tech-ECE Outstanding Junior Faculty Award (2002), the International Hybrid Microelectronics and Packaging Society Best Paper Award (1997), the Applied Computational Electromagnetics Society Best Paper Award (2001), and the IEEE International Conference on Microwave and Millimeter-Wave Technology Best Paper Award (2002). He was the 1999 Technical Program Co-Chair of the 54th ARFTG Conference and he is currently a member of the technical program committees of IEEE-IMS and IEEE-AP Symposiums. He is the Vice-Chair of IEEE-CPMT TC16 (RF Subcommittee). He is the Chair of the Atlanta section of IEEE MTT and AP societies. 